\title{
Robust Controller for a Full-Bridge Rectifier Using the IDA Approach and GSSA Modeling
}

\author{
Carlos Gaviria, Enric Fossas, and Robert Griñó, Member, IEEE
}

\begin{abstract}
An interconnection-and-damping assignment passivity-based control (IDA-PBC) for a full-bridge rectifier is presented in this paper. The closed-loop system performance fulfils unity power factor in the ac mains and output dc voltage regulation. The controller design takes advantage of the generalized state space averaging (GSSA) modeling technique to convert the quoted nonstandard problem (in actual variables) into a standard regulation one (in GSSA variables). In this approach, the output current is the measured signal instead of the line current; therefore, the number of sensors does not increase in comparison with traditional approaches. The whole system is robust with respect to load variations.
\end{abstract}

Index Terms-AC-DC converters, generalized state space averaging (GSSA), passivity-based control (PBC).

\section{INTRODUCTION}

I N INDUSTRIAL applications, dc power supplies connected to ac mains must have some desirable properties such as high power density, high power factor, high efficiency, low current distortion, and simple control scheme, particularly due to the enforcement of strict harmonic regulations (for example, IEC $61000-3-2$ ) [1]. For medium- and high-power applications, the most popular choice to this end is the power switching boost rectifier operating in continuous conduction mode [2]. However, it is difficult to control these converters because of their nonminimum phase behavior with respect to the output voltage, which, in turn, is worsened by the basic control objective of the sinusoidal shape in the converter line current. This specification has a nonstandard form because it only imposes the shape of a signal and not its value as a function of time, which would pose a tracking problem.

Using the generalized state space averaging (GSSA) [3] modeling method, it is possible to describe the control objectives, namely load voltage regulation and unity power factor at the input ac mains, as a regulation problem. Generally speaking, industrial loads for this rectifier are variable loads, this being the main drawback to obtain simple controllers. Common solutions require the sensing of input voltage, line current and output

Manucript received May 1, 2003; revised June 18, 2004. This work was supported in part by the Comisión Interministerial de Ciencia y Tecnología (CICYT) and by the European Comission under Project MOCOSHEV(DPI2002-03279) and Project GEOPLEX(IST-2001-34166). This paper was recommended by Associate Editor X. Yu.

C. Gaviria was with the Universitat Politècnica de Catalunya, Barcelona 08028, Spain. He is now with the Electronics Department, University of Cauca, Popayán, Colombia.

E. Fossas and R. Griñó are with the Institute of Industrial and Control Engineering, Universitat Politècnica de Catalunya, Barcelona 08028, Spain (e-mail: enric.fossas@upc.es; roberto.grino@upc.es).

Digital Object Identifier 10.1109/TCSI.2004.842881 voltage. Achieving robustness to load variations is not a simple control problem because whenever load varies, the amplitude of the line current must change to a new value to keep dc voltage regulation, but keeping the control objective over the line current shape. It is difficult to treat this problem as a tracking problem without measuring the load since the line current reference depends on it.

As reported in the excellent survey [4], traditional control strategies establish two loops: a line current inner loop for power factor compensation and an output voltage outer loop for voltage regulation. Works [5] and [6] warn of difficulties in actual implementations of these two loops because of the different dynamics they undergo. An adaptive scheme, which results in a complex control law, is proposed in [7]. All of these works use at least two sensors; even three as an extra output current measure is required to estimate the load.

The control proposed here has been designed for a non linear GSSA model with an inherited port-controlled Hamiltonian (PCH) structure. We take advantage of this structure to solve the aforementioned GSSA regulation problem and we obtain a closed-loop system robust to load variations. For this proposal, only two signals need to be measured, namely the output voltage and the output current, this being one of the main results of this work. The input voltage amplitude is supposed to be known, e.g., through sensing.

Interconnection-and-damping assignment passivity-based control (IDA-PBC) has been developed in the last years by Ortega et al. as a control technique based on energy balance. It was applied to solve regulation problems in physical systems written in Euler-Lagrange equations and recently extended to PCH systems. The reader is referred to [8] and the references therein for basic results.

The main contributions of the present work are: first, a tracking problem is solved in a PCH framework; using GSSA models it is possible to transform the nonstandard tracking control problem into a regulation one. Second, the extension of the Hamiltonian structure of the nonlinear model to the GSSA model and finally, the experimental validation of the control strategy designed.

The paper is organized as follows. Section II provides a short review of the GSSA modeling technique. Section III develops a preliminary analysis of the system based on the energy in steady state and states the control objectives. In Section IV, a GSSA model for the full-bridge converter in its relevant harmonics is obtained and written as a PCH system. Section V develops the IDA approach for this system to obtain a stabilising control law. Section VI shows simulation results obtained from a switched 
model of the full-bridge rectifier, and the robustness to load variations behavior is remarked. Section VII describes the experimental setup and shows experimental results. Finally, some conclusions are drawn.

\section{REVIEW OF GSSA METHOD}

The GSSA method was introduced in [3] and expanded later to highlight the accuracy of this modeling technique applied to describe the dynamic behavior of dc/dc converters in [9] and [10]. This review has been taken from the last reference.

The method is based on the fact that a signal $x(\tau)$ on the interval $\tau \in[t-T, t]$ can be represented by the Fourier series

$$
x(\tau)=\sum_{\ell=-\infty}^{\infty}\langle x\rangle_{\ell}(t) e^{j \ell \omega_{0} \tau}
$$

where $\omega_{o}=2(\pi / T)$ and $\langle x\rangle_{\ell}(t)$ are the time-dependent complex Fourier coefficients given by

$$
\langle x\rangle_{k}(t)=\frac{1}{T} \int_{t-T}^{t} x(\tau) e^{-j k \omega_{0} \tau} d \tau .
$$

To reconstruct $x(\tau)$ from its Fourier coefficients, (1) can be reformulated as

$$
x(\tau)=\langle x\rangle_{0}+2 \sum_{\ell=1}^{\infty}\left(\Re\langle x\rangle_{\ell} \cos \left(\ell \omega_{o} \tau\right)-\Im\langle x\rangle_{\ell} \sin \left(\ell \omega_{o} \tau\right)\right)
$$

where the time argument $t$ of $\langle x\rangle_{\ell}$ has been dropped to simplify the notation, and $\Re\langle x\rangle_{\ell}$ and $\Im\langle x\rangle_{\ell}$ are the real and imaginary parts of $\langle x\rangle_{\ell}$, respectively.

In order to use this representation for the $x(\tau)$ in a state-space model of a system, two useful facts concerning differentiation with respect to time and computation of the average of a product are

$$
\begin{aligned}
\frac{d\langle x\rangle_{k}(t)}{d t} & =\left\langle\frac{d x}{d t}\right\rangle_{k}(t)-j k \omega_{o}\langle x\rangle_{k}(t) \\
\langle q x\rangle_{k} & =\sum_{i=-\infty}^{\infty}\langle q\rangle_{k-i}\langle x\rangle_{i} .
\end{aligned}
$$

\section{Preliminary Analysis of System}

The following equations describe the dynamical behavior of the full-bridge boost type rectifier in Fig. 1:

$$
\begin{aligned}
& \frac{d \phi(t)}{d t}=\frac{-u(t)}{C} q(t)-\frac{r}{L} \phi(t)+v_{i}(t) \\
& \frac{d q(t)}{d t}=\frac{u(t)}{L} \phi(t)-i_{l}(t)
\end{aligned}
$$

where $\phi(t)$ is the magnetic flux through inductor $L, q(t)$ is the electrical charge in capacitor $C, r$ is a resistance modeling the parasitic resistive effect of the inductor and the switches, $u(t)$ describes the position of the switches taking values in the discrete set $\{-1,1\}, i_{l}(t)$ is the load current, and $v_{i}(t)=E \sin \left(\omega_{o} t\right)$ is the ac voltage source of amplitude $E$ and angular frequency $\omega_{o}=2 \pi f, f$ being the frequency in hertz.

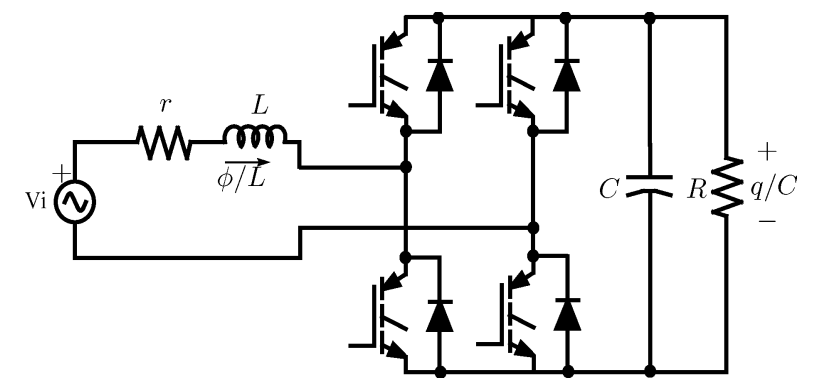

Fig. 1. Full-bridge boost type rectifier.

In this section, and only for GSSA modeling purposes, the load will be assumed resistive, then $i_{l}=(q / C)(1 / R)$.

As in [7], the control objectives for this rectifier are ${ }^{1}$

- The dc value of the output voltage $q(t) / C,\langle q(t)\rangle_{0} / C$ should be equal to a desired constant value $V_{d}>E$; i.e.,

$$
\langle q(t)\rangle_{0}{ }^{*}=C V_{d} .
$$

- The power factor of the converter should be equal to one. This means that, in steady state, the inductor current $\phi(t) / L$ follows a sinusoidal signal with the same frequency and phase as the ac-line voltage source; i.e.,

$$
\phi^{*}(t)=L I_{d} \sin \left(\omega_{o} t\right)
$$

where $I_{d}$ is the appropriate constant value fulfilling the aforementioned objective.

Note that the second control objective does not correspond to a tracking problem because amplitude $I_{d}$ depends on variable $i_{l}(t)$.

A useful variable transformation, which simplifies ${ }^{2}$ forthcoming developments, is obtained through $v(t)=-u(t) q(t)$ and $\mathbf{z}=\left[z_{1}, z_{2}\right]=\left[\phi(t),(1 / 2) q(t)^{2}\right]$. The system in the new variables is

$$
\begin{aligned}
& \frac{d z_{1}(t)}{d t}=-\frac{r z_{1}(t)}{L}+\frac{v(t)}{C}+v_{i}(t) \\
& \frac{d z_{2}(t)}{d t}=-\frac{v(t) z_{1}(t)}{L}-i_{l}(t) \sqrt{2 z_{2}(t)} .
\end{aligned}
$$

The energy in the storing elements $L$ and $C$ of this system can be described by

$$
H_{T}(t)=\frac{z_{1}(t)^{2}}{2 L}+\frac{z_{2}(t)}{C}
$$

and (9)-(10) can be rewritten as

$$
\begin{aligned}
{\left[\begin{array}{c}
\frac{d z_{1}}{d t} \\
\frac{d z_{2}}{d t}
\end{array}\right]=\left[\begin{array}{cc}
0 & v \\
-v & 0
\end{array}\right] } & \frac{\partial H_{T}}{\partial \mathbf{z}} \\
& -\left[\begin{array}{cc}
r & 0 \\
0 & C i_{l} \sqrt{2 z_{2}}
\end{array}\right] \frac{\partial H_{T}}{\partial \mathbf{z}}+\left[\begin{array}{c}
v_{i} \\
0
\end{array}\right]
\end{aligned}
$$

which corresponds to a PCHS system [8] of the form

$$
\dot{\mathbf{z}}=\left[\mathbf{J}_{T}(\mathbf{v})-\mathbf{R}_{T}(\mathbf{x})\right] \frac{\partial H_{T}}{\partial \mathbf{z}}(\mathbf{z})+\mathbf{g}_{T}
$$

\footnotetext{
${ }^{1}$ In this paper $*$ will be used to express the value in steady state

${ }^{2}$ First, the new input $v$ linearises and decouples the first equation; second, the Fourier expansion of $q^{2}$ is much simpler than that of $q$, at least in steady state.
} 
where $\mathbf{J}_{T}=-\mathbf{J}_{T}^{T}, \mathbf{R}_{T}(\mathbf{x})=\mathbf{R}_{T}^{T}(\mathbf{x}) \geq 0$ are the matrices in (12) describing the interconnection structure and damping, respectively. The last inequality results from $z_{2} \geq 0$ and $i_{l} \geq 0$ because the load voltage is non negative. The input voltage $\left(v_{i}, 0\right)$ is considered as an external disturbance modeled by vector $\mathbf{g}_{T}$. In order to obtain the simplest coherent GSSA model, let us determine the harmonic content of the states and the input in steady state.

To this end, let $z_{1}^{*}(t)=L I_{d} \sin \left(\omega_{o} t\right)$ be the $z_{1}$ desired dynamics and $i_{l}(t)$ the load current assuming a resistive load. In order to obtain the steady-state zero dynamics, let us take into account this assumption in (9)-(10) and let us solve for $v$ and $z_{2}$. The steady-state response yields

$$
\begin{aligned}
& z_{2}^{*}(t)=\alpha_{z 2}+\beta_{z 2} \sin \left(2 \omega_{o} t+\theta_{z 2}\right) \\
& v^{*}(t)=C\left(E-r I_{d}\right) \sin \left(\omega_{o} t\right)+I_{d} \omega_{o} L C \cos \left(\omega_{o} t\right)
\end{aligned}
$$

where

$$
\begin{aligned}
\alpha_{z 2} & =\frac{I_{d} R C^{2}}{4}\left(E-r I_{d}\right) \\
\beta_{z 2} & =\frac{I_{d} R C^{2}}{4} \sqrt{\frac{\left(E-r I_{d}\right)^{2}+\left(I_{d} \omega_{o} L\right)^{2}}{1+\left(\omega_{o} R C\right)^{2}}} \\
\tan \left(\theta_{z 2}\right) & =\frac{\left(E-r I_{d}\right)-\omega_{o} R C\left(\omega_{o} L I_{d}\right)}{\omega_{o} R C\left(E-r I_{d}\right)+\omega_{o} L I_{d}} .
\end{aligned}
$$

The value of parameter $I_{d}$ can be obtained from (7). Note that $\alpha_{z 2}$ is the output voltage dc component to be achieved. Thus, $\alpha_{z 2}=C^{2} V_{d}^{2} / 2$, and

$$
I_{d}=\frac{E}{2 r} \mp \sqrt{\left(\frac{E}{2 r}\right)^{2}-\frac{2 V_{d}^{2}}{r R}} .
$$

As in [2] and [7] the expression for the $I_{d}$ can also be obtained through power balance. The minus sign has been chosen since it yields a stable equilibrium point with lower power consumption. The total stored energy in steady-state results in

$$
H_{T}^{*}(t)=\alpha_{H}+\beta_{H} \sin \left(2 \omega_{o} t+\theta_{H}\right)
$$

where

$$
\begin{aligned}
\alpha_{H} & =\frac{I_{d} C R}{4}\left(E-r I_{d}\right)+\frac{L I_{d}^{2}}{4} \\
\beta_{H} & =\frac{\frac{I_{d} C R}{4}\left(E-r I_{d}\right)+\frac{L I_{d}^{2}}{4}}{\sqrt{1+\left(\omega_{o} R C\right)^{2}}} \\
\tan \left(\theta_{H}\right) & =\frac{1}{\omega_{o} R C} .
\end{aligned}
$$

Expressions (14), (15), and (8) show that a suitable GSSA model of the system, useful for controller design purposes, should contemplate the first harmonic Fourier components for $z_{1}(t)$, the zero and second harmonic Fourier components for $z_{2}(t)$ and the first harmonic Fourier components for $v(t)$. As for the Hamiltonian $H_{T}(t)$, from (17), the dc component and second harmonic should be considered. If, in addition, $C$ is chosen to obtain a low voltage ripple in the capacitor, then $\beta_{z 2}$ and $\beta_{H}$ are negligible with respect to $\alpha_{z 2}$ and $\alpha_{H}$, respectively.
Hence, the second harmonic Fourier components of $z_{2}(t)$ and $H_{T}(t)$ will not be considered from now on.

\section{FULl-BRIDGE RECTIFIER AS PCH SYSTEM IN GSSA VARIABLES}

Although the most general GSSA model of a system has infinite dimension, the harmonic contents of signals in steady state can be used to find accurately enough finite dimensional GSSA models. To this aim, using (4) and taking into account the Fourier components we have considered as relevant, the bilinear product $v(t) z_{1}(t)$ in (10) can be approximated as

$$
\begin{aligned}
\left\langle v z_{1}\right\rangle_{0} & =\sum_{k=-\infty}^{\infty}\langle v\rangle_{-k}\left\langle z_{1}\right\rangle_{k} \\
& =\langle v\rangle_{0}\left\langle z_{1}\right\rangle_{0}+2 \sum_{k=1}^{\infty}\left(\langle v\rangle_{k}^{R}\left\langle z_{1}\right\rangle_{k}^{R}+\langle v\rangle_{k}^{I}\left\langle z_{1}\right\rangle_{k}^{I}\right) \\
& \simeq 2\left(\langle v\rangle_{1}^{R}\left\langle z_{1}\right\rangle_{1}^{R}+\langle v\rangle_{1}^{I}\left\langle z_{1}\right\rangle_{1}^{I}\right) .
\end{aligned}
$$

Furthermore

$$
\left\langle i_{l} q\right\rangle_{0} \simeq\left\langle i_{l}\right\rangle_{0}\langle q\rangle_{0}+2\left(\left\langle i_{l}\right\rangle_{1}^{R}\langle q\rangle_{1}^{R}+\left\langle i_{l}\right\rangle_{1}^{I}\langle q\rangle_{1}^{I}\right) .
$$

As it has been assumed $q(t)$ has predominantly dc harmonic components, the complex coefficients of order one in (19) will be discarded. Thus, $\left\langle z_{2}\right\rangle_{0} \simeq(1 / 2)\left(\langle q\rangle_{0}\right)^{2}$ and $\langle q\rangle_{0} \simeq \sqrt{2\left\langle z_{2}\right\rangle_{0}}$. Hence, using (3), (18) and (19), the GSSA model of the system defined by (10) becomes

$$
\begin{aligned}
& \frac{d\left\langle z_{2}\right\rangle_{0}}{d t}=-\left\langle i_{l}\right\rangle_{0} \sqrt{2\left\langle z_{2}\right\rangle_{0}}-\frac{2}{L}\langle v\rangle_{1}^{R}\left\langle z_{1}\right\rangle_{1}^{R}-\frac{2}{L}\langle v\rangle_{1}^{I}\left\langle z_{1}\right\rangle_{1}^{I} \\
& \frac{d\left\langle z_{1}\right\rangle_{1}^{R}}{d t}=-\frac{r}{L}\left\langle z_{1}\right\rangle_{1}^{R}+\frac{1}{C}\langle v\rangle_{1}^{R}+\omega_{o}\left\langle z_{1}\right\rangle_{1}^{I} \\
& \frac{d\left\langle z_{1}\right\rangle_{1}^{I}}{d t}=-\frac{r}{L}\left\langle z_{1}\right\rangle_{1}^{I}+\frac{1}{C}\langle v\rangle_{1}^{I}-\omega_{o}\left\langle z_{1}\right\rangle_{1}^{R}-\frac{E}{2} .
\end{aligned}
$$

Let $\mathbf{x}=\left[\left\langle z_{2}\right\rangle_{0},\left\langle z_{1}\right\rangle_{1}^{R},\left\langle z_{1}\right\rangle_{1}^{I}\right], \mathbf{u}=\left[\langle v\rangle_{1}^{R},\langle v\rangle_{1}^{I}\right]$ be the state and control vectors, respectively, and

$$
\mathbf{x}^{*}=\left[\frac{C^{2} V_{d}^{2}}{2}, 0, \frac{-L I_{d}}{2}\right]
$$

the desired equilibrium.

The original control problem has become a regulation problem in the GSSA domain. For simplicity, let us denote the load current dc component by $I_{o}=\left\langle i_{l}\right\rangle_{0}$. Then, the system in (20) can be written as the PCH system:

$$
\begin{aligned}
{\left[\begin{array}{c}
\frac{d x_{1}(t)}{d t} \\
\frac{d x_{2}(t)}{d t} \\
\frac{d x_{3}(t)}{d t}
\end{array}\right]=} & {\left[\begin{array}{ccc}
0 & -u_{1} & -u_{2} \\
u_{1} & 0 & \frac{\omega_{o} L}{2} \\
u_{2} & -\frac{\omega_{o} L}{2} & 0
\end{array}\right] \frac{\partial H}{\partial \mathbf{x}} } \\
& -\left[\begin{array}{ccc}
C I_{o} \sqrt{2 x_{1}} & 0 & 0 \\
0 & \frac{r}{2} & 0 \\
0 & 0 & \frac{r}{2}
\end{array}\right] \frac{\partial H}{\partial \mathbf{x}}+\left[\begin{array}{c}
0 \\
0 \\
-\frac{E}{2}
\end{array}\right] .
\end{aligned}
$$

Or in a more compact form

$$
\dot{\mathbf{x}}=[\mathbf{J}(\mathbf{u})-\mathbf{R}(\mathbf{x})] \frac{\partial H}{\partial \mathbf{x}}(\mathbf{x})+\mathbf{g}
$$


where $\mathbf{J}=-\mathbf{J}^{T}, \mathbf{R}(\mathbf{x})=\mathbf{R}^{T}(\mathbf{x}) \geq 0$ are the interconnection-and-damping matrices, respectively, and vector $\mathrm{g}$ models an external disturbance. Note that $H(x)$ is the dc component of the Hamiltonian $H_{T}(z)$ in (11); i.e.,

$$
H(\mathbf{x})=\left\langle H_{T}(z)\right\rangle_{0}=\frac{1}{C}\left\langle z_{2}\right\rangle_{0}+\frac{1}{L}\langle\phi\rangle_{1}^{R^{2}}+\frac{1}{L}\langle\phi\rangle_{1}^{I^{2}}
$$

or

$$
H(\mathbf{x})=\frac{1}{C} x_{1}+\frac{1}{L} x_{2}^{2}+\frac{1}{L} x_{3}^{2} .
$$

The GSSA system in (22) preserves the PCH structure of the system in (12), with the remarkable advantage of a regulation control objective. This allows the IDA passivity based design approach to be methodically used. In this line, an IDA-PB control fulfilling system specifications is designed in the next section. The control law depends on the output voltage dc component and requires measuring the dc output current to guarantee robustness with respect to load variations.

\section{CONTROLler Design}

The final objective of the IDA-PBC approach [8] is to design a feedback control $\mathbf{u}=\boldsymbol{\beta}(\mathbf{x})$ such that the closed-loop dynamics is the PCH reference system

$$
\dot{\mathbf{x}}=\left[\mathbf{J}_{d}(\mathbf{x})-\mathbf{R}_{d}(\mathbf{x})\right] \frac{\partial H_{d}}{\partial \mathbf{x}}(\mathbf{x})
$$

where $\mathbf{J}_{d}(\mathbf{x})=-\mathbf{J}_{d}^{T}(\mathbf{x})$ and $\mathbf{R}_{d}(\mathbf{x})=\mathbf{R}_{d}^{T}(\mathbf{x}) \geq 0$ are targeted interconnection-and-damping matrices, and the new energy function $H_{d}(\mathbf{x})=H(\mathbf{x})+H_{a}(\mathbf{x})$ has a strict local minimum at the desired equilibrium.

Following [8], we proceed in the standard manner. (i)

1) Structure preservation. Given $\mathbf{J}_{d}(\mathbf{x})$ and $\mathbf{R}_{d}(\mathbf{x})$, let $\mathbf{J}_{a}(\mathbf{x})$ and $\mathbf{R}_{a}(\mathbf{x})$ be defined by

$$
\begin{aligned}
\mathbf{J}_{d}(\mathbf{x}) & :=\mathbf{J}(\mathbf{x}, \boldsymbol{\beta}(\mathbf{x}))+\mathbf{J}_{a}(\mathbf{x})=-\left[\mathbf{J}(\mathbf{x}, \boldsymbol{\beta}(\mathbf{x}))+\mathbf{J}_{a}(\mathbf{x})\right]^{T} \\
\mathbf{R}_{d}(\mathbf{x}) & :=\mathbf{R}(\mathbf{x})+\mathbf{R}_{a}(\mathbf{x})=\left[\mathbf{R}(\mathbf{x})+\mathbf{R}_{a}(\mathbf{x})\right]^{T} \geq 0
\end{aligned}
$$

Then, the desired dynamics is achieved if it is possible to find functions $\boldsymbol{\beta}(\mathbf{x})$ and $\mathbf{k}(\mathbf{x}):=\partial H_{a}(\mathbf{x}) / \partial \mathbf{x}$ satisfying

$$
\begin{aligned}
{\left[\mathbf{J}(\mathbf{x}, \boldsymbol{\beta}(\mathbf{x}))+\mathbf{J}_{a}(\mathbf{x})-\left(\mathbf{R}(\mathbf{x})+\mathbf{R}_{a}(\mathbf{x})\right)\right] \mathbf{k}(\mathbf{x}) } \\
=-\left[\mathbf{J}_{a}(\mathbf{x})-\mathbf{R}_{a}(\mathbf{x})\right] \frac{\partial H}{\partial \mathbf{x}}(\mathbf{x})+\mathbf{g} .
\end{aligned}
$$

2) Integrability. $\mathbf{k}(\mathbf{x})$ is the gradient of a scalar function. That is

$$
\frac{\partial k_{i}}{\partial x_{j}}(\mathbf{x})=\frac{\partial k_{j}}{\partial x_{i}}(\mathbf{x})
$$

\section{3) Equilibrium condition}

$$
\frac{\partial H_{d}}{\partial \mathbf{x}}\left(\mathbf{x}^{*}\right)=0 .
$$

4) Lyapunov Stability

$$
\left.\frac{\partial^{2} H_{d}}{\partial x^{2}}\right|_{\mathbf{x}^{*}}>0
$$

If conditions 1)-4) hold, then $\mathrm{x}^{*}$ is a (locally) stable equilibrium point of the closed-loop system.

Let us particularise the aforementioned procedure for the fullbridge boost rectifier controller defining $\mathbf{J}_{d}(\mathbf{x})=\mathbf{J}(\mathbf{x}, \boldsymbol{\beta}(\mathbf{x}))$ and $\mathbf{R}_{d}(\mathbf{x})=\mathbf{R}(\mathbf{x})$, i.e., $\mathbf{J}_{a}(\mathbf{x})=0$ and $\mathbf{R}_{\mathbf{a}}(\mathbf{x})=0$.

\section{A. Structure Preservation}

Equation (1) yields

$$
\begin{aligned}
& 0=-I_{o} C \sqrt{2 x_{1}} k_{1}-u_{1} k_{2}-u_{2} k_{3} \\
& 0=u_{1} k_{1}-\frac{r}{2} k_{2}+\frac{\omega L}{2} k_{3} \\
& 0=u_{2} k_{1}-\frac{\omega L}{2} k_{2}-\frac{r}{2} k_{3}+\frac{E}{2} .
\end{aligned}
$$

Then, from (28)-(29)

$$
\left.\begin{array}{l}
u_{1}=-\frac{-r k_{2}+\omega L k_{3}}{2 k_{1}} \\
u_{2}=\frac{\omega L k_{2}+r k_{3}-E}{2 k_{1}}
\end{array}\right\}
$$

\section{B. Integrability}

Replacing (30) in (27) and taking into account that $\mathbf{k}(\mathbf{x})=$ $\partial H_{a}(\mathbf{x}) / \partial \mathbf{x}$, the following partial differential equation is obtained:

$$
2 I_{o} C \sqrt{2 x_{1}}\left(\frac{\partial H_{a}}{\partial x_{1}}\right)^{2}=-r\left(\frac{\partial H_{a}}{\partial x_{2}}\right)^{2}-\left(r \frac{\partial H_{a}}{\partial x_{3}}-E\right) \frac{\partial H_{a}}{\partial x_{3}} .
$$

As we are interested in control inputs $u_{1}, u_{2}$, which only depend on the output voltage dc component, we take $k_{2}=k_{2}\left(x_{1}\right)$ and $k_{3}=k_{3}\left(x_{1}\right)$. Then, by the integrability condition

$$
\frac{\partial k_{i}}{\partial x_{1}}=\frac{\partial^{2} H_{a}}{\partial x_{1} \partial x_{i}}=\frac{\partial^{2} H_{a}}{\partial x_{i} \partial x_{1}}=0
$$

for $i=2,3$ and $k_{2}=a_{2}$ and $k_{3}=a_{3}$ are indeed constant. Thus, the PDE is actually an ODE on $x_{1}$, whose solution is given by

$$
H_{a}(\mathbf{x})=-\frac{2}{3} \sqrt{-\frac{\sqrt{2 x_{1}}}{I_{o} C} x_{1}\left(a_{2}^{2} r+a_{3}^{2} r-a_{3} E\right)}+a_{2} x_{2}+a_{3} x_{3}
$$

and

$$
\begin{aligned}
\mathbf{k}(\mathbf{x})= & {\left[\frac{\sqrt{2}\left(a_{2}^{2} r+a_{3}^{2} r-a_{3} E\right)}{6 \sqrt{-I_{o} C \sqrt{x_{1}} \sqrt{2}\left(a_{2}^{2} r+a_{3}^{2} r-a_{3} E\right)}}\right.} \\
& \left.-\frac{\sqrt{-I_{o} C \sqrt{x_{1}} \sqrt{2}\left(a_{2}^{2} r+a_{3}^{2} r-a_{3} E\right)}}{3 I_{o} C \sqrt{x_{1}}}, a_{2}, a_{3}\right] .
\end{aligned}
$$




\section{Equilibrium Assignmen}

From (31) and the definition of $H_{a}\left(H_{a}=H_{d}-H\right)$, the following conditions on $a_{2}, a_{3}$ and $I_{d}$ so that $\mathrm{x}^{*}$, from (21), is a singular point of $H_{d}$ are derived

$$
\begin{gathered}
\frac{1}{C}+\frac{\sqrt{-2 I_{o} C \sqrt{C^{2} V_{d}^{2}}\left(a_{2}^{2} r+a_{3}^{2} r-a_{3} E\right)}}{3 I_{o} C \sqrt{C^{2} V_{d}^{2}}} \\
=\frac{\sqrt{2}\left(a_{2}^{2} r+a_{3}^{2} r-a_{3} E\right)}{6 \sqrt{-I_{o} C \sqrt{C^{2} V_{d}^{2}}\left(a_{2}^{2} r+a_{3}^{2} r-a_{3} E\right)}} \\
a_{2}=0 \quad a_{3}-I_{d}=0 .
\end{gathered}
$$

This equations system has two solutions

$$
\left\{a_{2}=0, I_{d}=\frac{E+\sqrt{E^{2}-8 I_{o} V_{d} r}}{2 r}, a_{3}=I_{d}\right\}
$$

and

$$
\left\{a_{2}=0, I_{d}=\frac{E-\sqrt{E^{2}-8 I_{o} V_{d} r}}{2 r}, a_{3}=I_{d}\right\} .
$$

Then, taking the latter solution, $k_{1}$ and the control inputs derived in (30) are

$$
\begin{aligned}
& k_{1}=-\frac{\sqrt{2} \sqrt{I_{o}^{2} V_{d} C \sqrt{2} \sqrt{x_{1}}}}{2 I_{o} C \sqrt{x_{1}}} \\
& \left.\begin{array}{l}
u_{1}=-\frac{\omega L\left(-E+\sqrt{E^{2}-8 I_{o} V_{d} r}\right) C \sqrt{V_{o} V_{d}}}{4 r V_{d}} \\
u_{2}=
\end{array}\right\}
\end{aligned}
$$

where $V_{o}$ denotes the output voltage dc component $\left\langle v_{o}\right\rangle_{0}$ and $\sqrt{2 x_{1}}=\langle q\rangle_{0}=C\left\langle v_{o}\right\rangle_{0}$.

\section{Lyapunov Stability}

Replacing the values ${ }^{3}$ of $k_{2}, k_{3}$ and $I_{d}$ into $H_{a}$ and the last, in turn, into $H_{d}(\mathbf{x})$, the closed-loop Hamiltonian becomes

$$
\begin{aligned}
& H_{d}(\mathbf{x})=\frac{1}{6 C r L}\left(42^{(3 / 4)} \sqrt{C V_{d}} x_{1}^{(3 / 4)} r L-3 x_{3} C L E\right. \\
& \left.\quad+3 x_{3} C L \sqrt{E^{2}-8 I_{o} V_{d} r}-6 x_{1} r L-6 x_{2}^{2} C r-6 x_{3}^{2} C r\right) .
\end{aligned}
$$

Since the Hessian matrix of $H_{d}$ is diagonal with positive eigenvalues, $\mathrm{x}^{*}$, the singular point of $H_{d}$, is a local minimum. Moreover, $\mathbf{x}^{*}$ is an asymptotically stable equilibrium point as can be stated noticing in (26) that for $I_{o}>0, \mathbf{R}_{d}(\mathbf{x})=\mathbf{R}(\mathbf{x})$ is full rank, and the solution of $\partial H_{d} / \partial \mathbf{x}(\mathbf{x})=0$ is unique, namely $\mathbf{x}=\mathbf{x}^{*}$. If $I_{o}=0$, then $I_{d}=0$ and $x_{2}$ and $x_{3}$ converge to zero; then, $u_{2}=(E / 2) C \sqrt{V_{o} / V_{d}}$. Replacing this in (22) yields $d x_{3}(t) / d t=0$ if and only if $V_{o}=V_{d}$.

\section{Simulation Results}

\section{A. Linear Load}

The whole system behavior is simulated as a discrete control system using MatLab and Simulink. The continuous IDA-PB controller is discretised and implemented through a pulsewidth

${ }^{3}$ Note that taking $I_{o}=V_{d} / R$, the $I_{d}$ value obtained in (16) is recovered.

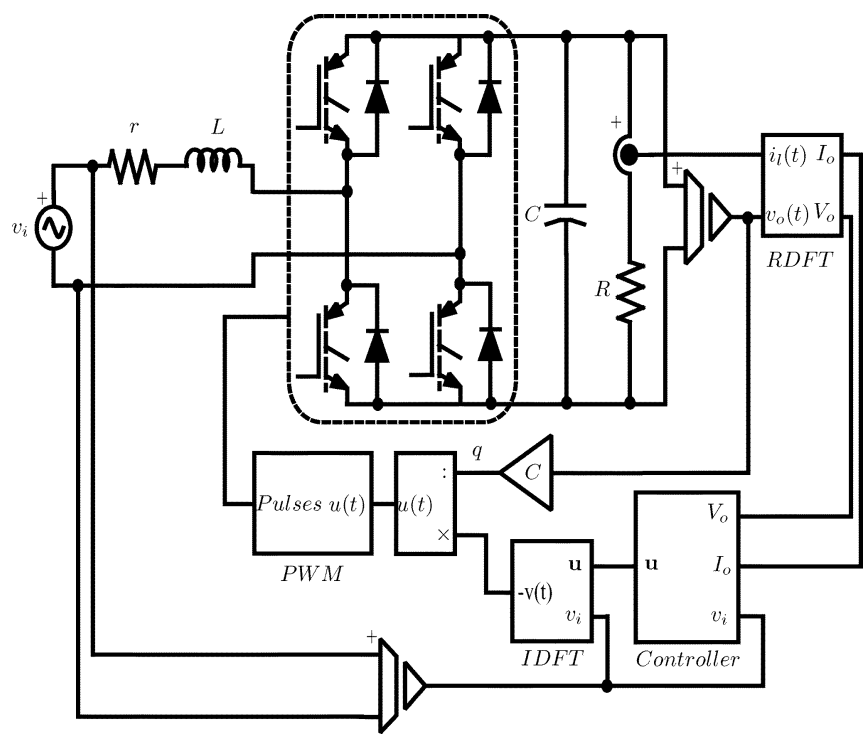

Fig. 2. General controller scheme.

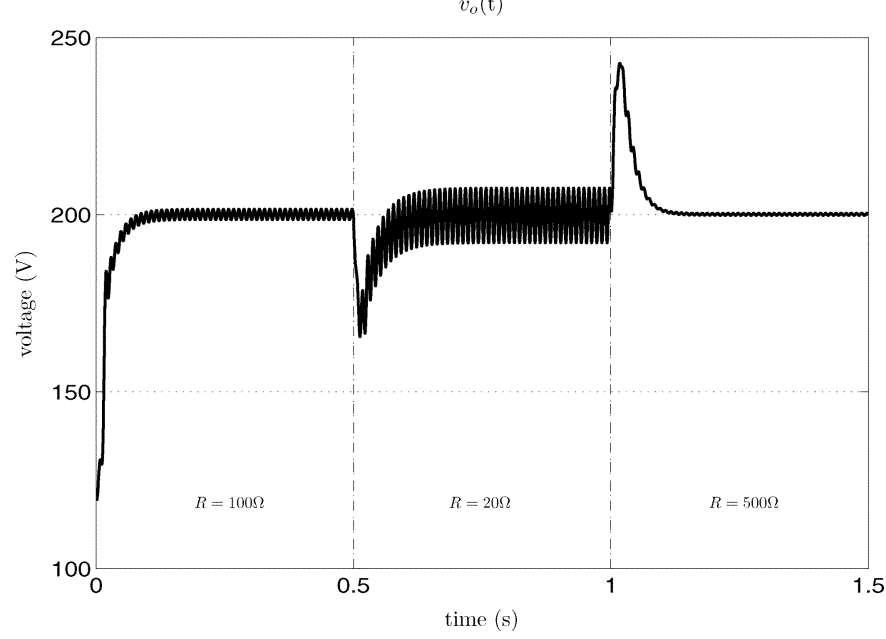

Fig. 3. Time evolution of dc output voltage $v_{o}(t)$ in front of load changes.

modulation (PWM) so that the simulations are closer to the actual system. Fig. 2 outlines the closed-loop diagram. The actual rectifier is shown at the top of the figure with Pulses as the input signal and the couple $i_{l}(t)$ and $v_{o}(t)$ as the output measured variables; note that the input voltage $v_{i}(t)$ is sensed too for being used as input in the Controller and inverse discrete Fourier transform (IDFT) blocks. The real-time discrete Fourier transform (RDFT) is carried out in the RDFT block. It is based on a recursive discrete Fourier transform technique, see [11] for details, which allows the right Fourier coefficients to be obtained at sampling times. The Controller block computes the suitable averaged Fourier components for the control signal $\mathbf{u}(t)$ while the IDFT is performed in the IDFT block to obtain the discrete $v(k T)$ control signal. Finally, the fixed frequency pulses are provided by the $P W M$ block by pulsewidth modulating the real valued $u(t)$ control signal.

The following parameters are used for simulation purposes: $R_{\text {nom }}=100 \Omega, L=2 \mathrm{mH}, C=2200 \mu \mathrm{F}, E=120 \mathrm{~V}$, 


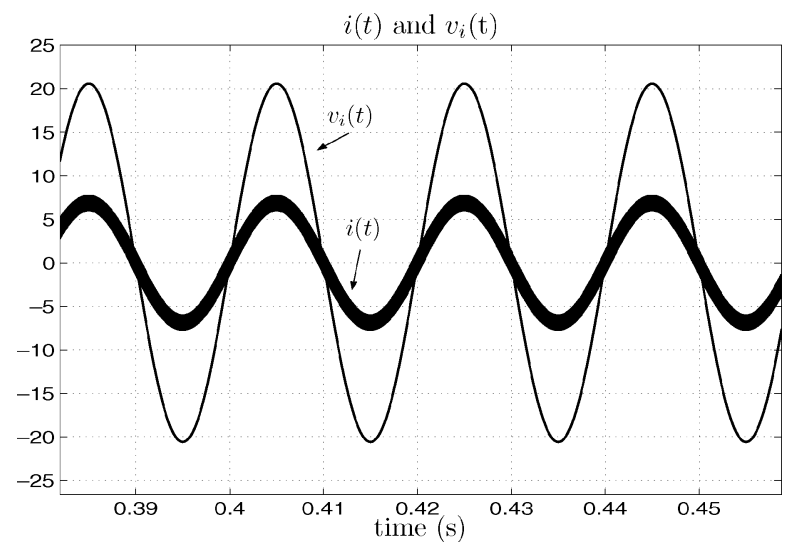

(a)

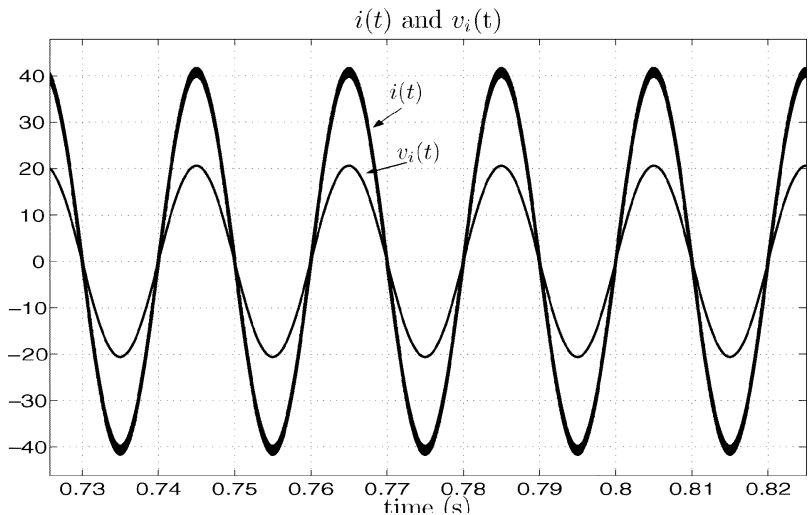

(b)

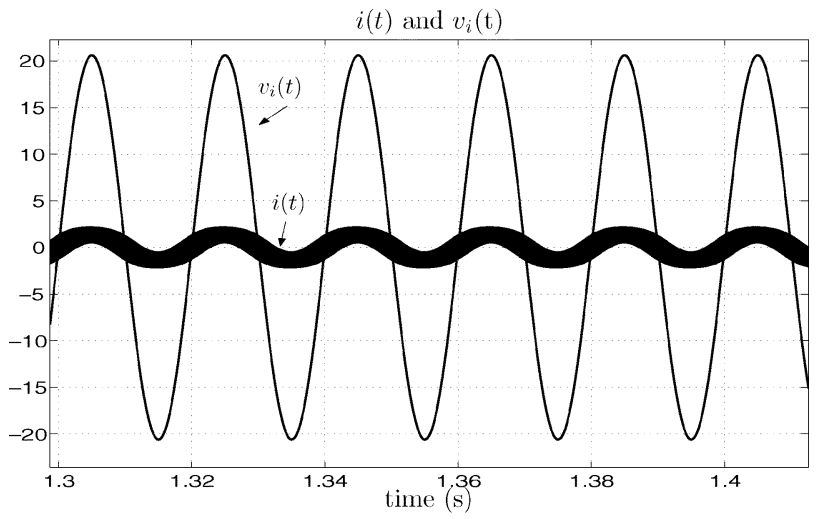

(c)

Fig. 4. $i(t)$ and scaled $v_{i}(t)$ : a) nominal load $R=100 \Omega$, b) $R=20 \Omega$, c) $R=50 \Omega$.

$f=50 \mathrm{~Hz}, V_{d}=200 \mathrm{~V}$; the PWM switching frequency is $f_{s}=20 \mathrm{kHz}$.

The obtained output voltage for the three values of the load, i.e., $R=R_{\text {nom }}=100 \Omega$ for $0 s<t<0.5 s, R=20 \Omega$ for $0.5 s \leq t<1 s$, and $R=500 \Omega$ for $1 s \leq t<1.5 s$, is depicted in Fig. 3. The inductor current $i(t)$ and the scaled input voltage $v_{i}(t)$ are depicted in Fig. 4 for each of the selected loads. These figures show that the specifications are fulfilled.

\section{B. Nonlinear Load}

Using averaged sensed variables instead of actual ones lets this controller to deal with nonlinear loads. To this end, simula-

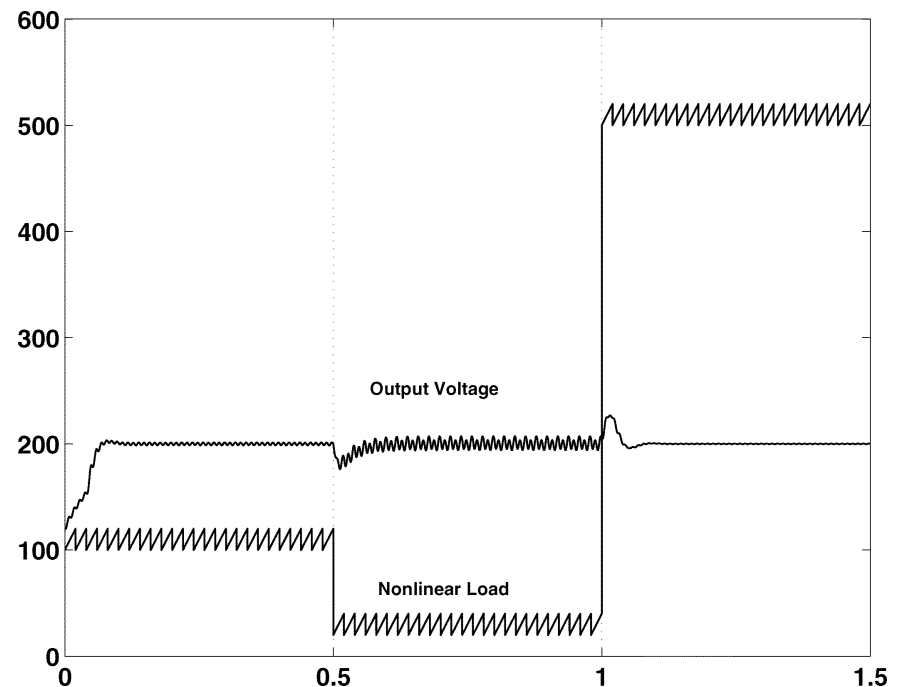

Fig. 5. DC bus voltage $\left(\mathrm{V}_{o}\right)$ and nonlinear load resistance $(\mathrm{R})$ values.

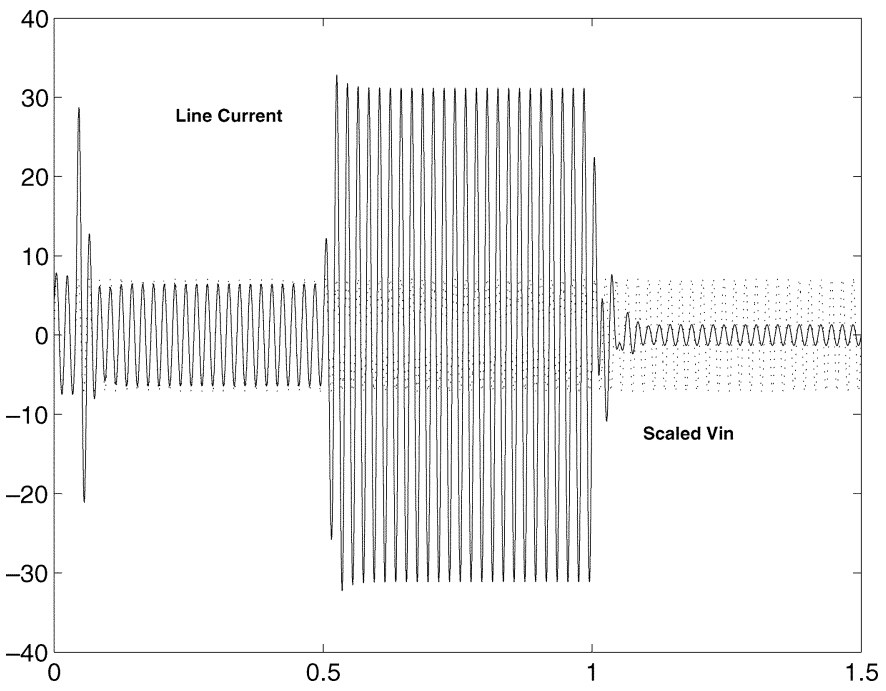

Fig. 6. Source voltage $v_{i}$ and line current $i_{l}$.

tion of this kind of load has been considered by means of resistance values composed by a mean value plus a triangular ripple (20\% amplitude) at the fundamental frequency. Fig. 5 shows the output dc voltage regulation performance and Fig. 6 shows the power factor correction performance for the case of a nonlinear load which mean values change from 100 to $50 \Omega$ and from the latter to $500 \Omega$.

\section{EXPERIMENTAL SETUP AND RESULTS}

The experimental setup used to test the designed controller has the following parts.

- Full-bridge boost converter with insulated-gate bipolar transistor (IGBT) switches (1200 V, $100 \mathrm{~A})$ and parameters: $r=0.092 \Omega, L=2.7 \mathrm{mH}, C=1400 \mu \mathrm{F}$. The switching frequency of the converter is $20 \mathrm{kHz}$ and a synchronous centered-pulse single-update PWM strategy is 


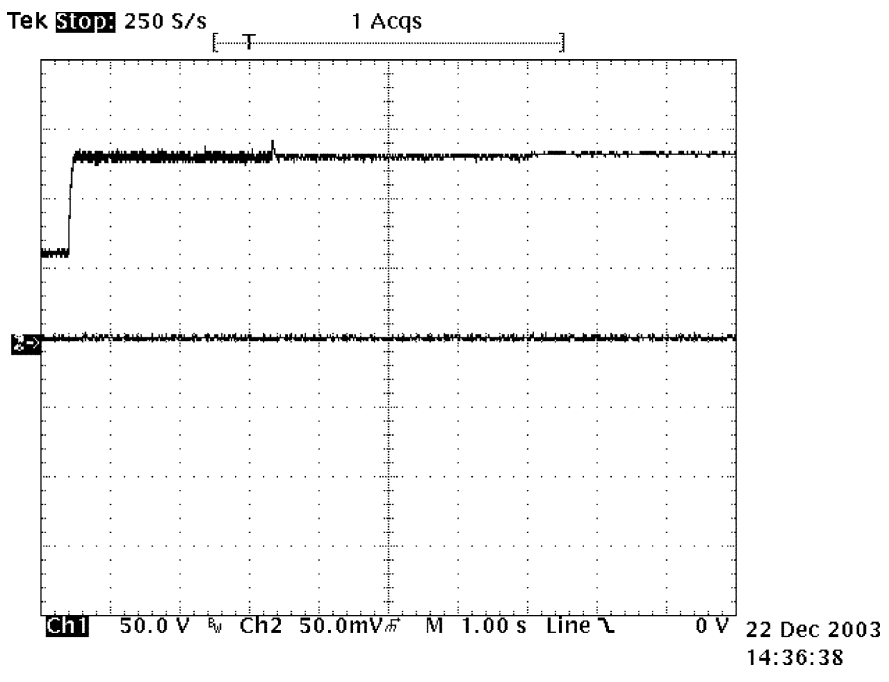

Fig. 7. DC bus voltage $\left(V_{o}\right)$ in front of load variations (1 s/time-division).

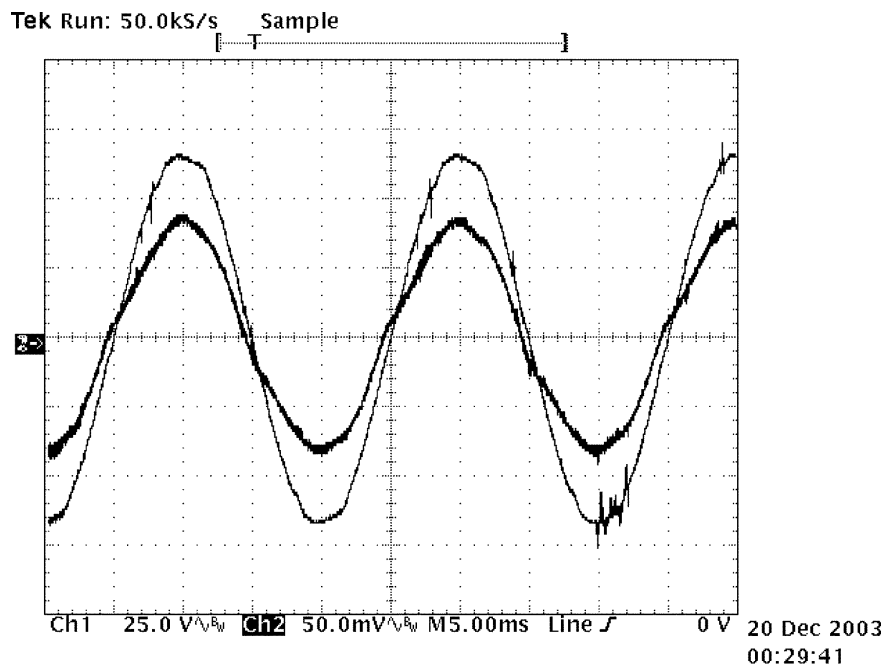

(a)

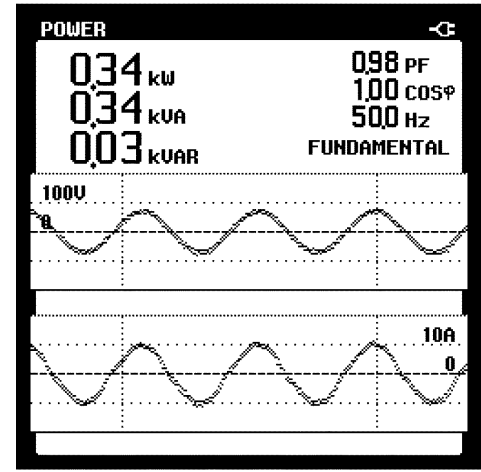

(b)

Fig. 8. Source voltage and line current, and power information at ac mains for $R=60 \Omega$. (a) $C H 1: v_{i} \mathrm{~V}, C H 2: i_{i} 10 \mathrm{mV} / \mathrm{A}$ (b) Power input data.

used to map the controller's output to the IGBT gate signals.

- Load: resistive load bank which allows to select $R=$ $513 \Omega, R=120 \Omega, R=60 \Omega$ or open circuit.

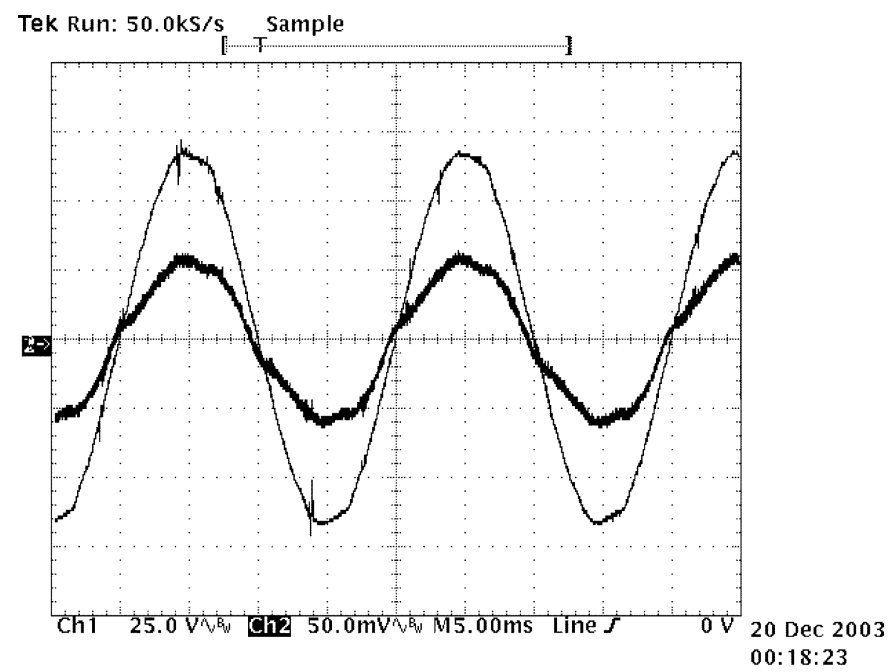

(a)

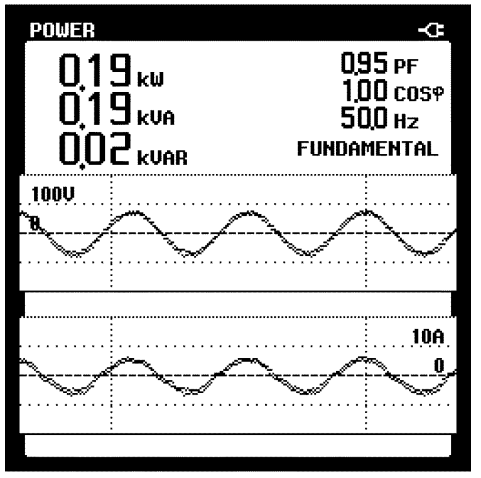

(b)

Fig. 9. Source voltage and line current, and power information at ac mains for $R=120 \Omega$. (a) $C H 1: v_{i} \mathrm{~V}, C H 2: i_{i} 10 \mathrm{mV} / \mathrm{A}$ (b) Power input data.

- Analog circuitry of sensors: the ac mains voltage, and dc bus voltage are sensed with isolation amplifiers whereas load current is sensed with an hall-effect sensor. All the signals from the sensors pass through the corresponding gain conditioning stages to adapt their values to analog-digital (A/D) converters.

- Control hardware and digital signal processing (DSP) implementation: the control algorithm has been implemented using the analog devices single-chip DSP motor controller ADMC401 processor. The processing core of this device is the 26-MHz fixed-point ADSP-2171 DSP processor. The ADMC401 deals with the PWM generation and the $\mathrm{A} / \mathrm{D}$ conversions. The sampling rate of the A/D channels is $T_{s}=20 \mathrm{kHz}$, the same as the switching frequency of the full-bridge system.

- The nominal rms ac mains voltage is $V_{s}=48.9 \mathrm{~V} \mathrm{rms}$ and its nominal frequency is $50 \mathrm{~Hz}$.

- Control objectives: The desired regulated dc output voltage and the power factor should be $V_{o}=130 \mathrm{~V}$ and near unity, respectively. It is important to note that the IGBT switches are oversized for this particular application resulting in undesirable power losses and harmonic distortion. These power losses has been taken into ac- 
count increasing the series resistance $r$ by a switch-loss resistance of $r s w=0.3 \Omega$. The system performance could be improved replacing these IGBT switches with low power ones.

From the aforementioned experimental setup some results have been obtained with the designed digital controller. These results are presented through oscilloscope and power analyzer screen dumps of the ac mains electrical variables and, as appropriate, the dc bus voltage.

\section{A. DC Bus Voltage Regulation}

In this experiment, at start time the dc bus voltage rests at the diode rectifier level with a resistive load of $R=60 \Omega$. Then, the control action is applied keeping the load resistance and the output voltage increases to the desired dc value. Afterwards, two load changes from $R=60 \Omega$ to $R=120 \Omega$ and from $R=120 \Omega$ to a no-load condition were applied at $t=3.3 \mathrm{~s}$ and $t=7.0 \mathrm{~s}$, respectively. Fig. 7 shows the shape of the dc bus output voltage. As it can be seen, the controlled system is robust with respect to load variations.

\section{B. Line Current and Power Factor Behavior}

This section shows, in Figs. 8 and 9, the line current shape and power factor value for two load conditions, namely $R=$ $60 \Omega$ and $R=120 \Omega$. As it can be seen, the higher the power managed, the closer to unity the power factor is. It is important to remark that the $\cos (\phi)$ is equal to one in both cases.

\section{CONCLUSION}

This paper shows how the GSSA modeling technique can be used not only to accurately model the behavior of variables in an ac-dc full-bridge power electronic converter, but also to explore advanced control techniques taking advantage of the inherent domain change, from time to GSSA domain.

In the case considered here, a nonstandard tracking control problem for a full-bridge boost rectifier results in a regulation one. An IDA-PB control has been designed measuring the load current and the load voltage, and presuming the input voltage is known. The closed-loop system is robust to load variations achieving unity power factor in the ac mains and load voltage regulation. Some experimental results are included showing the the feasibility of the designed controller.

\section{REFERENCES}

[1] B. Lin and H. Lu, "Single-phase power-factor-correction ac/dc converters with three pwm control schemes," IEEE Trans. Aerosp. Electron. Syst., vol. 36, no. 1, pp. 189-200, Jan. 2000.

[2] R. Griñó, E. Fossas, and D. Biel, "Sliding mode control of a full-bridge unity power factor rectifier," in Proc. Nonlinear Adaptive Control, vol. 281, Nov. 2001, pp. 139-148.

[3] S. Sanders, J. Noworolski, X. Liu, and G. Verghese, "Generalized averaging method for power conversion systems," IEEE Trans. Power Electron., vol. 6, no. 4, pp. 251-259, Apr. 1991.

[4] O. García, J. Cobos, R. Prieto, P. Alou, and J. Uceda, "Power factor correction: A survey," in Proc. IEEE 32nd Annu. Power Electronics Specialists Conf. (PESC.'01), vol. 1, 2001, pp. 8-13.
[5] D. Lee, G. Lee, and K. Lee, "Dc-bus voltage control of three-phase ac/dc pwm converters using feedback linearization," IEEE Trans. Ind. Appl., vol. 36, no. 3, pp. 826-832, May/Jun. 2000.

[6] R. Morici, C. Rossi, and A. Tonielli, "Variable structure controller for ac/dc boost converter," in Proc. IEEE 20th Int. Conf. Industrial Electronics, Control Instrumentation, vol. 3, 1994, pp. 1449-1454.

[7] G. Escobar, D. Chevreau, R. Ortega, and E. Mendes, "An adaptive passivity-based controller for a unity power factor rectifier," IEEE Trans. Control Syst. Technol., vol. 9, no. 4, pp. 637-644, Jul. 2001.

[8] R. Ortega, A. Schaft, B. Maschke, and G. Escobar, "Interconnection and damping assigment passivity-based control of port-controlled Hamiltonian systems," Automatica, vol. 38, pp. 585-596, 2002.

[9] J. Mahdavi, A. Emaadi, M. Bellar, and M. Ehsani, "Analysis of power electronic converters using the generalized state-space averaging approach," IEEE Trans. Circuits Syst. I, vol. 44, no. 8, pp. 767-770, Aug. 1997.

[10] V. Caliscan, G. Verghese, and A. Stanković, "Multi-frequency averaging of dc/dc converters," IEEE Trans. Power Electron., vol. 14, no. 1, pp. 124-133, Jan. 1999.

[11] J. Rosendo and A. Gómez, "Efficient moving-window dft algorithms," IEEE Trans. Circuits Syst. II, Analog Digit. Signal Process., vol. 45, no. 2, pp. 256-260, Feb. 1998.

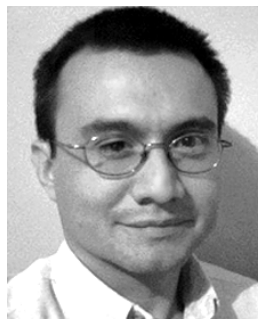

Carlos Gaviria received the electronic engineering degree from the University of Cauca, Popayán, Colombia, and the Ph.D. degree in automatic control from the Universitat Politècnica de Catalunya, Barcelona, Spain, in 1993 and 2004, respectively.

Since 1997, he has been an Assistant Professor in the Electronic Department, University of Cauca. His research interests include nonlinear control and control of power electronics converters.

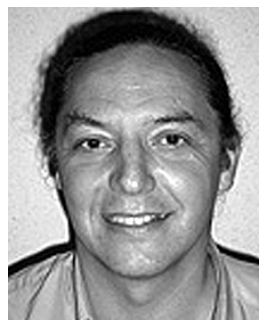

Enric Fossas received the M.S. and Ph.D. degrees in mathematics from Barcelona University, Barcelona, Spain, in 1981 and 1986, respectively.

Since 1981, he taught mathematics at the Barcelona University and mathematics and automatic control at the Universitat Politècnica de Catalunya, Barcelona, Spain. He is currently an Assistant Professor in the Department of Automatic Control and Computer Engineering, Universitat Politècnica de Catalunya, where he is also with the Institute of Industrial and Control Engineering. His research interests include nonlinear control (theory and applications), particularly variable Structure systems, with applications to switching converters. $\mathrm{He}$ is a member of SIAM.

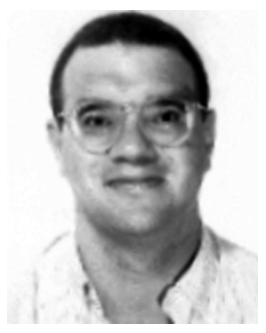

Robert Griñó (M'99) received the M.Sc. degree in electrical engineering and the Ph.D. degree in automatic control from the Universitat Politècnica de Catalunya (UPC), Barcelona, Spain, in 1989 and 1997, respectively.

From 1990 and 1991, he was a Research Assistant at the Instituto de Cibernética,UPC. From 1992 to 1998, he was an Assistant Professor in the Systems Engineering and Automatic Control Department, Institute of Industrial and Control Engineering, Barcelona, where he has been an Associate Professor since 1998. His research interests include digital control, sensitivity theory, nonlinear control and control of power electronics converters.

Dr. Griñó is an Affiliate Member of IFAC. 